\title{
A Hard X-Ray Telescope Science Enhancement Package for the Constellation X-Ray Mission
}

\author{
Brian Ramsey ${ }^{1}$ and Paul Gorenstein ${ }^{2}$ \\ ${ }^{1}$ Space Science Office, NASA/ Marshall Space Flight Center, Huntsville, Al 35812 \\ ${ }^{2}$ Harvard-Smithsonian Center for Astrophysics, Cambridge, MA 02138
}

\begin{abstract}
1. ABSTRACT
Details of a hard-X-ray science enhancement package for the Constellation-X mission are presented. A scientific case is made for the inclusion of such an instrument on the planned mission and a detailed design is presented that will satisfy science requirements yet fall within the ground rules for enhancement packages: a cost of less than $\$ 100 \mathrm{M}$ and a mass of no more than $100 \mathrm{~kg}$.
\end{abstract}

\section{INTRODUCTION}

The instrument described here was proposed in response to the Constellation-X solicitation of "white papers" containing concepts for the Science Enhancement Package (SEP) that will augment the capability of the mission ${ }^{1}$. The SEP is required to be compatible with a redesigned spacecraft and launch vehicle that are smaller than before. The premise is that the effective area and bandwidth of the soft-x-ray telescope (SXT) will remain essentially the same as in the original design. Any additions should add no more than $100 \mathrm{~kg}$ to the mass of the payload and no more than $\$ 100 \mathrm{M}$ to the cost.

The motive behind this proposal is the concern that the bandwidth of the SXT is not sufficiently broad to accomplish one of the most important scientific objectives of the Const ellation X-Ray Mission: obtaining unambiguous measurements of the profile of a $\mathrm{K} \alpha$ fluorescence line of iron that has been redshifted into a broadly extended feature by strong gravity forces in the vicinity of an accreting black hole. The proposed instrument is a hard-x-ray telescope (HXT) that extends the bandwidth of Con-X and permits measurements of the magnitude and functional form of the continuum on which the Fe feature sits. The continuum spectrum in the vicinity of the Fe line may be influenced by the "Compton hump" that is present in the X-ray spectrum of a black hole. The SXT will not be able to detect it over an energy range that is sufficiently large to define its functional form because the Compton hump reaches a peak at an energy beyond its high energy cutoff.

\section{SCIENTIFIC JUSTIFICATION}

Observing and quantifying the gravitational redshift in the vicinity of black holes is a major, if not the most important, scientific objective of Con-X as this will provide new information about the behavior of matter in strong gravitational fields and the laws of general relativity. The nearby $(z=0.00775)$ Seyfert galaxy MCG-6-30-15 contains a prime example of a gravitationally-broadened Fe line. The abundance of iron is 2 to 3 times solar ${ }^{2}$, which makes it a particularly good object from which to draw conclusions about the broad Fe feature. The broad-line effect was discovered by ASCA ${ }^{3}$ and has been observed since by ASCA again on several occasions, as well as BeppoSAX ${ }^{4}$ and RXTE $^{5}$. Furthermore, there was a long XMM-Newton exposure ${ }^{6}$ and a more recent Suzaku observation ${ }^{7}$. In ${ }^{8}$, several models were considered including the spin of the black hole that could explain the shape of the broad Fe line. 
A figure in the Suzaku paper illustrates why it is desirable to have good data at $20 \mathrm{keV}$ and above for interpreting the spectrum. Figure 1 is the energy spectrum of $M C G-6-30-15$ relative to a function that varies as $E^{-2}$. The points at energies up to $12 \mathrm{keV}$ are data from the CCD detectors (XIS) and the higher energy points, which extend to about 40 $\mathrm{keV}$ were obtained by a combination of a PIN diode and a GSO crystal scintillator.

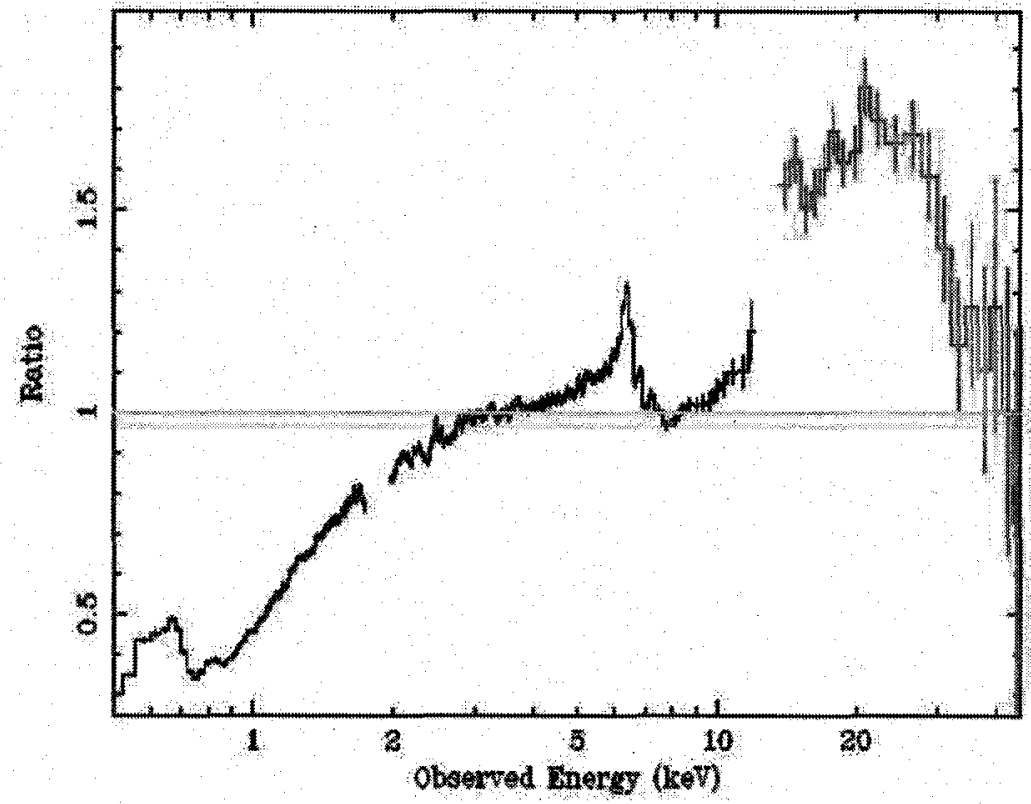

Fig. 1: Energy spectrum relative to the function $E^{2}$ of the Seyfert galaxy MCG-6-30-15 as seen by Suzaku. This figure is a reproduction of Fig. 4 of paper?

Between the Compton hump whose effects appear to extend down to the region of the Fe line, the broadened $\mathrm{Fe}$ line, and the absorption at low energy, the energy range where the ratio of the observed spectrum to a power law continuum is constant is very small. Consequently there is considerable ambiguity concerning the isolation of the broadened Fe line from the continuum and what its exact shape is.

Figure 2 is reconstructed from the Suzaku data shown in Figure 1. The Suzaku data points (circles) are the ratios with respect to a continuum function that varies as $\mathrm{E}^{-2}$. Two other sets of simulated data points are shown: one is obtained by changing the amplitude of the $\mathrm{E}^{-2}$ reference function by $2 \%$ (crosses) and the other by changing the power law reference function by $5 \%$ to $\mathrm{E}^{-2.1}$ (squares). Differences among the three sets of points illustrate the ambiguity of determining the precise shape of the gravitationally-broadened Fe line and how dependent that is upon the knowledge of the continuum. Figure 1 suggests that the influence of the Compton hump persists down to $7 \mathrm{k} \mathrm{eV}$ and perhaps even lower. Con-X will certainly observe many other objects with the goal of studying the gravitationally-broadened $\mathrm{Fe}$ line. These observations may include cases where the Compton hump has even greater influence upon the continuum in the region of the red shifted Fe line. 


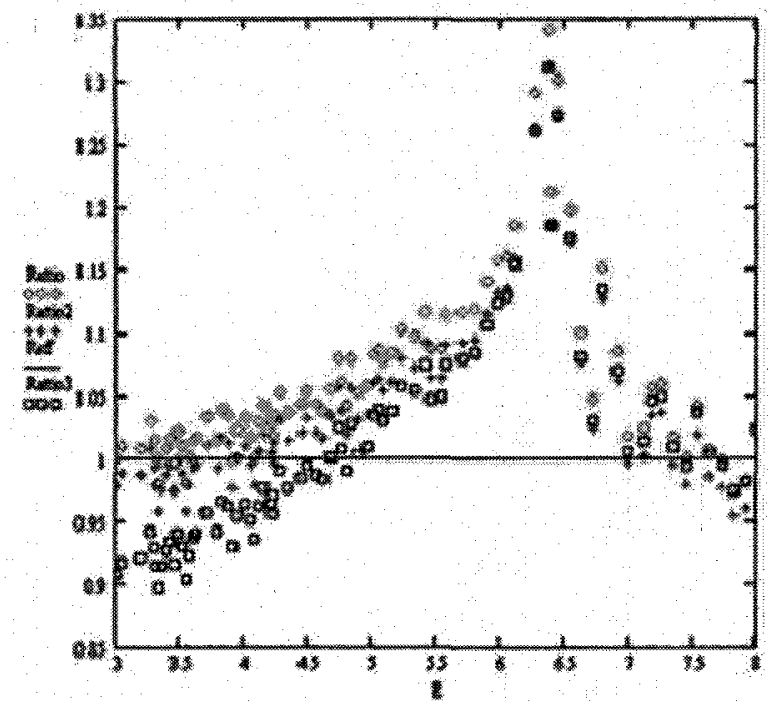

Fig. 2: Suzaku data points that appear in Fig. 2 of paper $^{7}$ are reproduced here without the error bars (red circles). Two other sets of data points are shown, blue crosses and black squares, based upon slightly different underlying continuum spectra (see text).

A recent XMM-Newton publication ${ }^{9}$ contains a figure, which is reproduced below in part (Figure 3 ), that shows the presence of broadened Fe lines in eight Seyfert galaxies. In each case the data have larger errors than the MGC-6-3015 data but one can see that the Fe line profile varies considerably from one object to another. This is not surprising given that the black holes at the center of each Seyfert do not all have the same mass and spin. Most cases exhibit significant changes in the continuum with respect to a power law spectrum with a constant spectral index even below $10 \mathrm{keV}$. In addition a very strong correlation between the intrinsic spectral slope in X-rays and the amount of Compton reflection in Seyfert AGNs has been reported ${ }^{10}$.
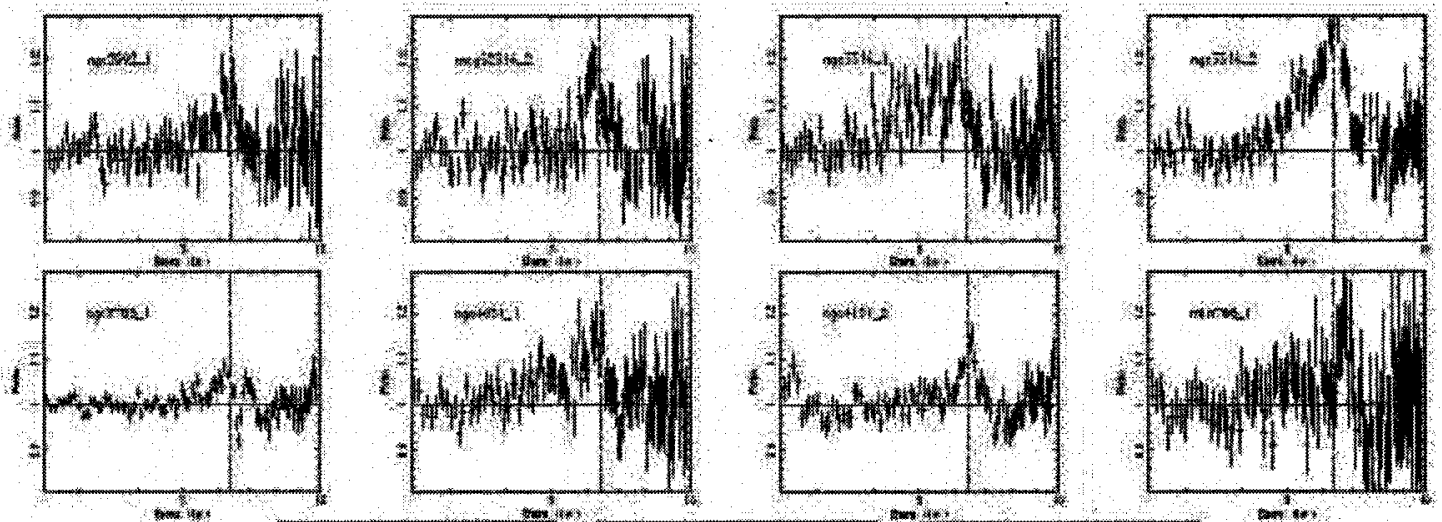

Fig. 3: Spectra of eight Seyfert galaxies relative to a power law reference as observed by XMMNewton (taken from a figure that appears in ${ }^{9}$ ).

There is no doubt the evidence for a gravitationally-redshifted Fe is supported by observations of ASCA, Suzaku, and XMM-Newton. However, in all cases the error bars are large and one can have doubts that the contribution of the continuum to the spectrum below $6.4 \mathrm{keV}$ has been taken into account accurately. Con-X will no doubt be able to reduce the error bars significantly and measure the energy profile of the redshifted Fe line plus continuum much more 
precisely. (Thanks to the high spectral resolution of the detectors Con-X will also be able to detect for the first time absorption edges and absorption lines in the profile.) However, in order to subtract the continuum and isolate the Fe line profile unambiguously it will be necessary to observe the continuum and Compton hump over an energy band that extends beyond the high energy cutoff of the SXT.

Furthermore, we note that Con-X will be a national observatory devoted to X-ray spectroscopy for use by many observers. They will be pursuing a wide variety of science objectives that will be enabled by or can benefit from extending the bandwidth of Con-X. Non-thermal processes, e.g. synchrotron radiation and inverse Compton scattering are factors in radiation from AGN jets and highly magnetized neutron stars. Non -thermal X-rays are usually more prominent at higher energy than the current limit of the SXT. Studies involving non-thermal X-rays will reap large benefits from extending the bandwidth of Con-X. Because background is reduced by orders of magnitude a focusing telescope operating in the 15 to $40 \mathrm{keV}$ band will have orders of magnitude more sensitivity in pointed measurements than all previous instrument.

\section{TECHNICAL DESCRIPTION}

\subsection{Overview}

The purpose of the proposed hard X-ray telescope system for a Con-X Science Enhancement Package is to extend the bandwidth beyond the high-energy cutoff of the SXT. The concept is based upon technology developed and tested for the original Con-X HXT that was removed in the recent mission re-design. The difference is the telescope modules are each lighter weight and there are fewer, two as compared to the original twelve. Our HXT concept utilizes multilayer-coated nickel optics coupled with cadmium-zinc-telluride focal plane detectors, optimized to provide a significant increase in collecting area above $15 \mathrm{keV}$.

The proposed optics are manufactured using a replication process in which thin nickel shells are electroformed on super-polished aluminum masters (mandrels) from which they are later released by differential thermal expansion. The resulting full-shell optics are inherently stable and deliver good angular resolution performance when simply held in locating spiders at their two ends. The electroformed-nickel-replication (ENR) process has been used very successfully on previous missions to fabricate the telescopes for Swift a nd XMM-Newton.

MSFC has an ENR development program that has produced mirror shells up to a factor of four times thinner than the thinnest shells used on XMM. Test shells, 100-microns-thick, and of diameters 15 and $23 \mathrm{~cm}$ have been coated with multilayers at SAO and mounted by colleagues at the Brera Observatory, Italy (Figure 4). They were tested in UV light at Brera and with X-rays at the Panter facility in Germany. To date, better than 30 arcsec HPD has been demonstrated and that is mostly limited by the mounting process. A new mounting and alignment system, currently under development at MSFC, promises better performance. Our performance goal is 20 arcsec HPD for mounted shells. Details of the fabrication, coating, and testing appears in various publications ${ }^{11,12}$ and the alignment system is

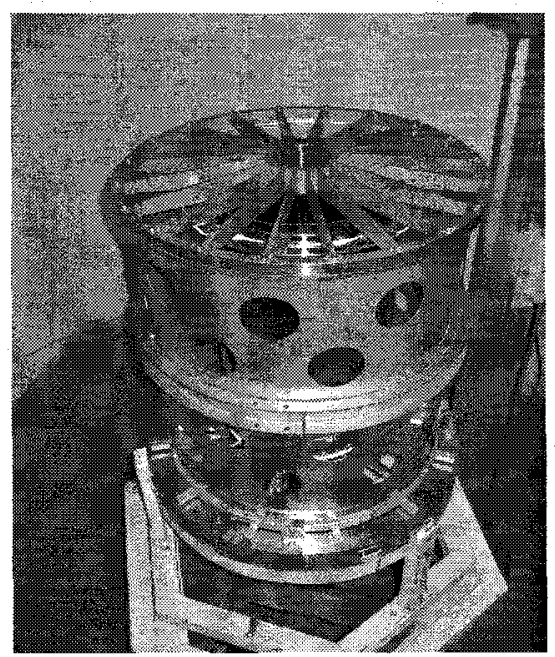

Fig. 4: Two 100-micron-thick test mirror shells installed in structure prior to testing at the Panter facility. 
described elsewhere in this volume ${ }^{13}$.

According to the SXT model's predictions it has an effective (system) area of $240 \mathrm{~cm}^{2}$ at $15 \mathrm{keV}$, dropping to just 41 $\mathrm{cm}^{2}$ at $20 \mathrm{keV}$. The function of the hard X-ray telescopes is to extend the energy band past $15 \mathrm{keV}$. Both iridium and multilayer coatings can be employed to provide significant effective area at high-energy. For example, the 23-cm mirror shell of the telescope shown in Figure 4 was coated with a W/Si multilayer whose period was graded with depth, while the $15-\mathrm{cm}$ mirror was coated with iridium. The coating assignments of the mirrors determine the energy band. Greater use of multilayer coatings favors effective area at the higher energy end of the band at the expense of the effective area at lower energy. Figure 6 shows the effective area of one possible set of (all multilayer) coatings. There is a wide range of possibilities for the Con-X Facility Science Team or other scientific committees to select the best science-driven effective area requirements.

For the focus of the above mirror modules pixellated detector arrays based on the room temperature semiconductor compound Cadmium-Zinc-Telluride (CZT) is proposed. These have high efficiency and good energy resolution in the energy range of interest and are well developed ${ }^{14}$. This is the type of detector selected for the original HXT.

\subsection{Configuration}

A two-module configuration is proposed for the enhancement package that maximizes the required collecting area function within the given SEP weight budget. The modules are located outboard of the SXT optics in either the three or four SXT configurations under consideration, as shown in Figure 5 below for the 4 optic case. The 4-SXT option places the HXT detector close to, but not outside, the edge of the detector bench. Note that in this SXT configuration there is ample room inside the central mirror bench area to accommodate both HXT optics, but some documents show SXT-detector-related hardware in the central detector bench area, which could preclude its use for the HXT detectors.

The two identical mirror modules each consist of 68 nested nickel-alloy shells ranging in diameter from $15 \mathrm{~cm}$ to 34 $\mathrm{cm}$. The shells are $60 \mathrm{~cm}$ total length and are a monolithic structure conta ining both ' $\mathrm{P}$ ' and ' $\mathrm{H}$ ' segments. Note that up to 10 extra shells can be added per module if the mass contingency allocated is not used.

\section{HXT GEOMETRY}

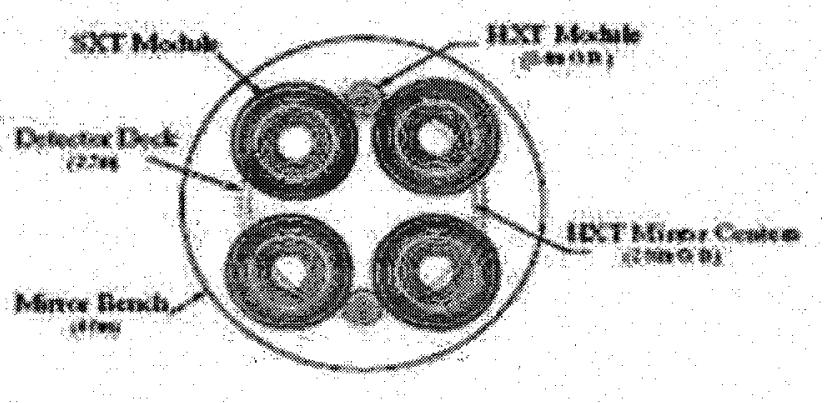

Fig. 5: Location of HXT telescopes, the 2small (red) circles.. SXT mirrors are the 4 larger(blue) circles.
The shells are fabricated from a high-strength nickel alloy and are 100 micron thick out to 25 $\mathrm{cm}$ diameter, gradually increasing to 150 microns thick at $34 \mathrm{~cm}$. A composite housing, matched to the thermal expansion coefficient of nickel contains the shells which are held in place by a pair of diamond turned spiders, fabricated in steel. The outer diameter of each mirror module, including thermal insulation, is $40 \mathrm{~cm}$, and the full module length is $66 \mathrm{~cm}$.

The proposed focal plane detectors are based on those developed for the HEFT and NuSTAR programs ${ }^{15}$. These are pixellated cadmium-zinctelluride devices with custom readout chips and have high quantum efficiency and excellent energy resolution ( $<1 \mathrm{keV}$ FWHM) over the bandwidth of interest. The pixel size is well matched to our optics: a 500-micron-resolution detector oversamples a 10-m-focal length, 20 arcsec HPD optic by a factor of two, leading to an overall system resolution of 22 arcsec. Total detector power ( 2 units) is expected to be around $12 \mathrm{~W}$, including detector heaters, but excluding mirror thermal control. 
For estimating the overall detector dimensions and weights we have based our concept on the device developed for the NuSTAR SMEX payload ${ }^{16}$. The envelope for each detector unit, $15.0 \mathrm{~cm}$ diameter including active shields and thermal insulation, is estimated to be just inside the detector bench in the 4 SXT config uration shown in Figure 5 .

Mass numbers, see Table 1 below, are derived from the calculated weights of the mirror shells in the configuration described above and the housing mass is scaled from those previously used in the HERO balloon payload. The mass of each detector system is based upon that previously generated for Con-X. and the NuSTAR experience.

Table 1: Estimated Total Mass for 2 HXT Telescopes

\begin{tabular}{|l|l|}
\hline Item & Mass (kg) \\
\hline & \\
\hline Shells & 29.6 \\
\hline Housings + blanket & 6.75 \\
\hline Detectors & 7.7 \\
\hline Sub-total per telescope & 44.1 \\
\hline Central electronics unit & 2.7 \\
\hline Contingency (10\%) & 9.0 \\
\hline & \\
\hline Total & $\mathbf{9 9 . 8}$ \\
\hline
\end{tabular}

\subsection{Performance} configuration.

Figure 6, below, shows the total system effective area as a function of energy for the proposed two-telescope

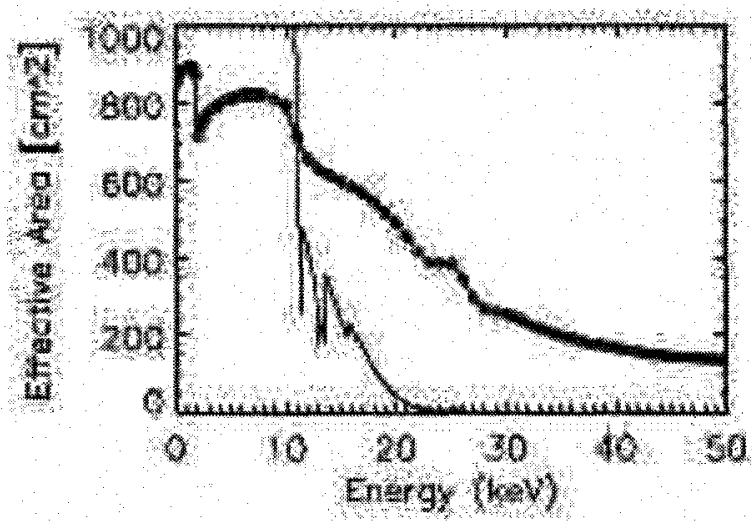

Fig. 6: Effective area (upper curve) of the proposed 2-telescope HXT as a function of energy. The lower curve represents the SXT area in the 4 telescope configuration.

The expected ( $5 \mathrm{~s})$ continuum sensitivity $(\mathrm{dE} / \mathrm{E}=50 \%$ ) has been calculated for the HXT configuration with this total effective area. Figure 7 shows this as a function of energy for two integration times, $10^{5} \mathrm{sec}$ and $10^{6} \mathrm{sec}$. The background for this calculation was taken from a detailed simulation of an HXT reference configuration in an L2 orbit $^{17}$. Note that most of the detector weight in the above table is in the BGO/CsI anticoincidence shields. Lighter shields could be investigated (such as plastic scintillators plus a mod est amount of passive shielding); the small focal spot of the 22 arcsec optics helps in this regard as a factor of two increase in background at $20 \mathrm{keV}$ only degrades the $10^{5} \mathrm{sec}$ sensitivity by $10 \%$. 
Fig. 7: Continuum sensitivity of the proposed 2-telescope configuration. Shown are (5-sigma) curves for $10^{5}$ and $10^{6} \mathrm{sec}$

http:/constellation.gsfe.nasa.gov/

2 Fabian, A. C., Vaughan, S., Nandra, K., Iwasawa, K., Ballantyne, D. R., Lee, J. C., De Rosa, A., Turner, A. Young, A. J., A long hard look at MCG-6-30-15 with XMM-Newton, MNRAS 335, L1, 2002.

${ }^{3}$ Tanaka, Y., Nandra, K., Fabian, A. C., Inoue, H., Otani, C., Dotani, T., Hayashida, K., Iwasawa, K., Kii, T., Kunieda, H., Gravitationally Redshifted Emission Implying an Accretion Disk and Massive Black-Hole in the Active Galaxy MCG:-6-30-15, Nature 375, 659, 1995.

${ }^{4}$ Guainazzi, M., Matt, G., Molendi, S., Orr, A., Fiore, F., Grandi, P., Matteuzzi, A., Mineo, T., Perola, G. C., Parmar, A. N., Piro, L., BeppoSAX confirms extreme relativistic effects in the X-ray spectrum of MCG-6-30-15, A \& A. 341, L27, 1999 .

5 Vaughan, S. and Edelson, R., Evidence for Rapid Iron Ka Line Flux Variability in MCG-6-30-15 ApJ 548, 694, 2001. 
${ }^{6}$ Wilms J. Reynolds, C. S., Begelman, M. C. Reeves. J., Molendi S., Struber, R., and Kendziorra, E., XMM-EPIC observation of MCG-6-30-15: direct evidence for the extraction of energy from a spinning black hole? MNRAS 328, L27, 2001.

${ }^{7}$ Miniutti, G., Fabian, A., Anabuki, N., Crummy, J., Fukazawa, Y., Gallo, L., Haba, Y., Hayashida, K., Holt, S., Kunieda, H., and 14 coauthors, Suzaku Observations of the Hard X-Ray Variability of MCG -6-30-15: the Effects of Strong Gravity around a Kerr Black Hole, 2006, PASJ (in press). Also astro-ph 0609521.

${ }^{8}$ Brenneman, L. W., Reynolds, C. S., Constraining Black Hole Spin via X-Ray Spectroscopy, Ap.J. 652,1028.

${ }^{9}$ Nandra, K, O'Neil, P. M., George, I. M., Reeves, J. N. and Turner, T. J., An XMM-Newton survey of broad iron lines in $A G N$, Astron. Nachr. 999, 789, 2006.

${ }^{10}$ Zdziarski, A. A., Lubinski, P., and Smith, D. A., Correlation between Compton reflection and X-ray slope in Seyferts and X-ray binaries, MNRAS 303, L11, 1999.

${ }^{11}$ Ramsey, B., R Elsner, D. Engelhaupt, M. Gubarev, J. Kolodziejczak, S. O'Dell, C. Speegle and M. Weisskopf, “ The development of hard-x-ray optics at MSFC," SPIE 5168, 129, 2003.

${ }^{12}$ Romaine, S., et. al., "Development of a prototype nickel optic for the Constellation-X hard $x$-ray telescope: III (Proceedings Paper)", SPIE 5900, 59, 2005.

${ }^{13}$ Gubarev' M., Arnold, W., Benson, C., Kester, T., Lehner, D., Ramsey, B. and Upton, R., "Mounting and Alignment of Full-Shell Replicated X-Ray Optics," this volume.

${ }^{14}$ Chen, C., Cook, W., Harrison, F., Lin, J., Mao, P. and Schindler, S., “ Characterization of the HEFT CdZnTe pixel detectors," SPIE 5198, 9, 2004.

${ }^{15} \mathrm{http}: / /$ www.nustar.caltech.edu/

${ }^{16}$ Fiona Harrison/Caltech: private communication.

${ }^{17}$ Armstrong, T.W., Colborn, B.L. and Ramsey, B.D., "Initial estimates of radiation backgrounds for the hard-x-ray telescope on the planned Constellation-X Mission," SAIC-TN-99015R2, 1999. 